\title{
Innate Immune Control of Salmonella enterica Serovar Typhimurium: Mechanisms Contributing to Combating Systemic Salmonella Infection
}

\author{
Mary Jo Wick \\ Department of Microbiology and Immunology, Institute of Biomedicine, University of Gothenburg, \\ Gothenburg, Sweden
}

\section{Key Words}

Salmonella $\cdot$ Innate immunity $\cdot$ Dendritic cell $\cdot$ Monocyte $\cdot$ Gut-associated lymphoid tissue

\begin{abstract}
Infections with Salmonella enterica serovars remain a serious problem worldwide. While serovar Typhi causes significant morbidity and mortality that is restricted to humans, serovar Typhimurium causes gastroenteritidis in humans and can also infect other animals. As mice with the susceptible Nramp1 locus get systemic infection with serovar Typhimurium, murine infection models using this serovar have been widely used to decipher the immune mechanisms required to survive systemic Salmonella infection. This review summarizes recent studies in murine infection models that have advanced our understanding of the events that occur during the first days after oral Salmonella infection. The pathways of bacterial penetration across the intestinal epithelium, bacterial spread to draining (mesenteric) lymph nodes and dissemination to systemic tissues is discussed. The response of myeloid cell populations, including dendritic cells, inflammatory monocytes and neutrophils, during the early stage of infection is also discussed. Finally, the mechanisms driving recruitment of myeloid cells to infected intestinal lymphoid tissues and what is known about Toll-like receptor signaling pathways in innate immunity to Salmonella infection is also discussed.

Copyright $\odot 2011$ S. Karger AG, Basel
\end{abstract}

\section{KARGER}

Fax +4161306 1234

E-Mail karger@karger.ch

www.karger.com
2011 S. Karger AG, Basel

$1662-811 \mathrm{X} / 11 / 0036-0543 \$ 38.00 / 0$

Accessible online at:

www.karger.com/jin

\section{Introduction}

Infection of humans and animals with the facultative intracellular pathogen Salmonella remains a serious global health problem. For example, the human-restricted serovars of Salmonella enterica, Typhi and Paratyphi, cause typhoid fever that claims several hundred thousand lives every year. This systemic disease, which is transmitted by contaminated food and water, is characterized by high fever, diarrhea and hepatosplenomegaly. Life-threatening complications of intestinal hemorrhage and perforation can arise and lead to sepsis, which is fatal if not treated. Nontyphoid serovars of Salmonella are also responsible for significant morbidity and mortality worldwide. Although gastroenteritis caused by nontyphoid serovars remains localized in healthy individuals, who typically recover without treatment, these infections can be fatal for immune-compromised individuals including those with HIV. Nontyphoid serovars also cause serious invasive diseases in children in developing countries.

Although Salmonella infection can be treated with antibiotics, these are often not readily available in endemic areas and antibiotic resistance is on the rise. Vaccines against typhoid fever do exist, but they exhibit only moderate protection in adults and are ineffective in children, and vaccines against nontyphoid Salmonella serovars currently do not exist. Overall, new and improved vaccines against Salmonella are clearly warranted. An important aspect of developing vaccines is to understand the pathogenesis of the infecting microbe. Indeed, infec- 
tion models in mice are invaluable tools in the vaccine development process. In the case of Salmonella, infection of mice that have the susceptible allele of Slc1la1 (Nramp-1) with serovar Typhimurium causes a systemic infection that is widely used to study the pathogenesis of and immunity to this bacterium. Although this mouse model, like any nonprimate model used to study human diseases, is not perfect, it has indeed provided valuable information regarding Salmonella infection. A clear advantage of murine models is that they allow mechanistic aspects of immunity to infection to be systematically studied. Indeed, such studies have contributed greatly to our understanding of the host response required to clear Salmonella infection, from the events that occur in the innate response to limit bacterial replication through development of adaptive immunity that ultimately eradicates the infection. This review will focus on the early events that occur during Salmonella infection to minimize bacterial growth and set in motion the adaptive immune response. The data discussed are from studies with $S$. enterica serovar Typhimurium in murine infection models and highlights recent findings. For the sake of brevity, S. enterica serovar Typhimurium is referred to as Salmonella in the remainder of this review.

\section{The First Step of Salmonella Infection: Bacterial Penetration into Host Tissues}

Physical barriers and resident immune cells in the intestine comprise the first line of defense against infection by orally acquired pathogens such as Salmonella. An important physical barrier preventing ingested bacteria from accessing host tissue is the single epithelial cell layer that separates the intestinal lumen from the underlying cells in the lamina propria. Several routes may contribute to Salmonella penetration across the intestinal epithelial layer [for review, see 1]. However, bacteria crossing the epithelium overlying Peyer's patches via M cells is a predominant pathway, particularly for invasive (pathogenicity island-1-proficient) bacteria. Bacterial crossing via $M$ cells overlying solitary intestinal lymphoid tissues (SILTs), which are organized lymphoid tissue in the intestine other than Peyer's patches, also contributes to bacterial entry [2]. Finally, non-M cell-mediated 'cell sampling' routes whereby Salmonella can access the lamina propria have also been described $[1,3]$. This pathway involves predominantly invasion-deficient Salmonella and is mediated by $\mathrm{CX}_{3} \mathrm{CR}^{+}$phagocytes $[1,3]$. Eloquent studies have greatly advanced our understanding of the function of
$\mathrm{CX}_{3} \mathrm{CR}^{+}$phagocytes [for review, see 4] and interestingly, the $\mathrm{CX}_{3} \mathrm{CR}^{+}$phagocytes involved in luminal sampling do not migrate to the mesenteric lymph nodes (MLN) that drain the intestine [5]. This suggests that bacterial transit mediated by $\mathrm{CX}_{3} \mathrm{CR} 1^{+}$phagocytes may play a role in bacterial destruction rather than $\mathrm{T}$ cell priming. Alternatively, $\mathrm{CX}_{3} \mathrm{CR}^{+}$phagocytes may pass bacteria or bacterial antigens to dendritic cells (DCs) for subsequent presentation to $\mathrm{T}$ cells. Thus, although the cell sampling pathway involving $\mathrm{CX}_{3} \mathrm{CR}^{+}$phagocytes can contribute to penetration of invasion-deficient Salmonella into the lamina propria [3], M cell-mediated pathways dominate for invasion-proficient Salmonella.

Once invasive Salmonella use M cells to cross the epithelial barrier, the bacteria encounter resident cells in the subepithelial dome. This region is located beneath $\mathrm{M}$ cells in Peyer's patches and SILTs, and is rich in DCs, particularly of the $\mathrm{CD} 8 \alpha^{-}$subset $[1,6]$. Moreover, although other myeloid lineage cells such as macrophages and neutrophils can be found in low numbers in Peyer's patches of naive mice using sensitive, high-throughput techniques such as flow cytometry $[7,8]$, cells expressing Gr-1 and F4/80 are not readily detected in the subepithelial dome by immunohistochemistry $[1,9]$. Thus, the organization of Peyer's patches, with DCs being locally enriched in the subepithelial dome, allows these cells ready access to bacteria that penetrate via $\mathrm{M}$ cells. Given the pivotal role of DCs in priming naive $T$ cells to start an immune response, DCs in intestinal lymphoid tissues are important players in initiating immunity to oral bacterial pathogens.

\section{Dissemination of Orally Acquired Salmonella to MLN and Other Tissues}

Once Salmonella penetrate the intestinal epithelial layer after oral infection they seed the intestinal lymphoid organs, Peyer's patches and SILTs, and the lamina propria. They also spread to the MLN draining the intestine, and to extraintestinal tissues, particularly the spleen and liver [2, 10-12]. Although much progress has been made regarding how Salmonella crosses the intestinal epithelium and establishes infection in the immediate target organs, our understanding of the mechanism by which Salmonella progresses to the MLN and extraintestinal tissues has lagged behind. However, progress in the field of migratory DCs $[5,13]$ and direct data addressing the dissemination of orally acquired Salmonella [12] have provided insight into this area. For example, Voedisch et al. [12] showed that colonization of the spleen and liver by 
invasive Salmonella occurs by a mechanism distinct from colonization of MLN. In particular, increasing mobilization of intestinal DCs using a TLR agonist or a growth factor for DCs (FLT3L) increased Salmonella in the MLN but not the spleen or liver. Likewise, reducing CCR7-mediated DC emigration from the intestine via lymph by orally infecting CCR7-deficient mice reduced Salmonella in the MLN but not the liver or spleen [12]. However, some invasive Salmonella still reached the MLN in the absence of CCR7-mediated transport. This indicates that there are complimentary, CCR7-independent mechanism(s) that can disseminate invasive Salmonella from the intestine to the MLN. Thus, the present picture of Salmonella dissemination supports an important role for CCR7-dependent bacterial transport from the intestine to the MLN via DCs migrating in lymph, while alternate pathway(s) allow the bacteria to reach systemic tissues. What are the pathway(s) that allow Salmonella to rapidly access extraintestinal tissues without necessarily relying on transport to the MLN in lymph-borne DCs? A possibility is that intestinal bacteria in phagocytes can directly access the blood, which can rapidly seed systemic tissues $[14,15]$. Finally, data support an important protective role for MLN in limiting the spread of intestinal bacteria, both commensals [16] and pathogens [12], to systemic tissues. Thus, recent findings show specialized roles of intestinal cells and lymphoid tissues in controlling the dissemination of luminal bacteria, and that different processes dominate the colonization of the MLN and systemic tissues after oral Salmonella infection.

\section{Dendritic Cell Responses to Salmonella Infection}

The innate immune response is rapidly activated after oral infection, and significant changes in cell number are apparent within 2 days of intragastric Salmonella administration $[7,17]$. Changes that occur include recruitment of DCs, inflammatory monocytes and neutrophils. Although DCs accumulate in Peyer's patches in response to infection, their increase is relatively modest [11]. Given the unique role of DCs in priming T cells and their limited role in keeping bacterial replication in check, it is likely that their functional features rather than their number per se is most important. For example, DCs expressing CCR6 are rapidly recruited to the sub epithelial dome region, and these cells have a key role in activating Salmonella-specific T cells [18]. In addition to the slight increase in DC number, another important aspect of the early DC response to infection is their maturation. This process, which involves changes in DCs as detected phenotypically by increased surface expression of MHC class II and the co-stimulatory molecules CD86, CD80 and CD40, is necessary for efficient activation of naive T cells [19]. Given the role of intestinal DCs in maintaining intestinal homeostasis [20], the maturation process of intestinal DCs, perhaps coupled with recruitment of new 'nontolerized' DCs, is particularly important for their ability to prime naive $T$ cells to orally acquired pathogens $[19,20]$. Indeed, DCs harbor Salmonella after oral infection $[11,21]$ and are critical to priming bacteria-specific $\mathrm{T}$ cells [18]. Thus, although recruitment of CD11chi DCs to Peyer's patches during Salmonella infection is modest, the accumulation of particular subsets, such as DCs expressing CCR6 and/or cells less conditioned in a tolerogenic environment, may be important [20]. The initiation of DC maturation is an essential early event in the response to Salmonella infection and is a key step in starting an adaptive immune response to this pathogen.

Interestingly, Salmonella preferentially induces death of one subset of CD11 $c^{\text {hi }}$ conventional DCs during infection, the $\mathrm{CD} 8 \alpha^{+}$subset, while $\mathrm{CD} 8 \alpha^{-}$DCs and recruited inflammatory monocytes are resistant [22]. This bacteria-induced DC death depends on MyD88, an adaptor protein that controls signaling through many Toll-like receptors (TLRs), and on signaling through TNFR1. DCs have a fairly short life span of a few days, which could serve as a general mechanism to minimize undesired conditions such as chronic lymphocyte activation or autoimmunity. However, DCs must survive long enough to activate $\mathrm{T}$ cells, and an insufficiently short life span negatively influences the magnitude of the $\mathrm{T}$ cell response. What could be the consequences of infection-induced DC death? Is this a virulence factor aimed to undermine adaptive immunity and reduce the host's ability to clear the infection? Does it promote or impede bacterial spread? The answers to these questions are not clear, but we do know that conventional DCs are required to activate Salmonella-specific T cells in vivo [18]. The CD $8 \alpha^{+}$DC subset also has some special features of its antigen presentation capacity. For example, CD $8 \alpha^{+}$DCs efficiently take up dead cells, cross-present antigens on MHC-I and produce IL-12p70 and thus influence CD4 ${ }^{+}$Th1 T cell responses [23]. As IFN- $\gamma$-producing $\mathrm{CD}^{+}{ }^{+} \mathrm{Th} 1 \mathrm{~T}$ cells are critical to surviving Salmonella infection, [24], selective death of this subset could be a way for bacteria to avoid clearance. In this respect, it is also intriguing to note that recruited inflammatory monocytes have a limited capacity to process Salmonella for antigen presentation [7, 22]. However, CD8 $\alpha^{-}$DCs from infected mice have the capac- 
ity to present Salmonella antigens on MHC-II for CD $4^{+}$ $\mathrm{T}$ cell recognition [22]. The consequence of Salmonellainduced death of $\mathrm{CD} 8 \alpha^{+}$DC during infection is not known. However, it could be a way for Salmonella to slow down effective antigen presentation and dampen an effective Th1 $\mathrm{T}$ cell response, which may favor bacterial replication and spread within an infected host.

\section{Dendritic Cell Maturation during Infection}

As mentioned above, DCs in the steady-state intestine have an important role in maintaining tolerance to intestinal flora and potential food antigens, and the process of maturation is important in overcoming this tolerogenic bias. Infection with intestinal pathogens must therefore trigger DC maturation. How is DC maturation triggered in vivo? We know that an important initiating event occurs when conserved microbial structures, so-called microbe-associated molecular patterns (MAMPs) such as LPS and flagella, engage pattern recognition receptors on host cells. Indeed, host cells express different pattern recognition receptor families that recognize MAMPs, which act as common denominators indicating an infection. The receptor families involved in sensing bacterial infection include C-type lectin receptors, nucleotide-binding oligomerization domain-like receptors (NLRs) and TLRs [2527]. Distinct functions of these receptor families create a complimentary approach to detecting and controlling infections $[25,28]$. For example, C-type lectin receptors often act as phagocytic receptors while NLRs are intracellular sensors of microbial components, particularly peptidoglycan structures, and also detect cell injury [25, 27]. Ligand recognition by NLRs results not only in production of cytokines and chemokines, but also in assembly of inflammasomes that results in production of the active forms of IL-1 $\beta$ and IL-18 $[25,29]$. The inflammasome is indeed important in the innate immune response to Salmonella $[29,30]$. Recent reviews on NLR signaling and the inflammasome are available, and the reader is referred to these for more detailed discussion of these topics [25, 29].

TLRs are also important in controlling Salmonella infection [31]. A main function of TLRs, which are expressed by intestinal epithelial cells and immune cells, is to initiate signaling cascades that lead to pro-inflammatory cytokine and chemokine production by NF- $\kappa \mathrm{B}$ activation [26]. Several of the cytokines induced by bacterial triggering of pattern recognition receptors, particularly IL-1 $\beta$ and TNF- $\alpha$, can influence MHC-II and costimulatory molecule expression and contribute to DC maturation during
Salmonella infection $[11,21]$. However, phenotypic maturation as assessed by altered expression of MHC and costimulatory molecules does not necessarily reflect functional maturation of the DCs. That is, full DC maturation must result in DCs that elicit effector functions in T cells (i.e. IFN- $\gamma$ production) [32]. An important component of achieving fully functional DC maturation is signals delivered by direct DC interaction with microbial structures [32]. In contrast, signals delivered indirectly by cytokines in the absence of direct microbial contact results in phenotypic but not necessarily functional DC maturation [32]. Studies to decipher the contribution of direct versus indirect (cytokine-mediated) maturation of DC during Salmonella infection, which is biologically more complex relative to studies injecting purified bacterial products, have been performed [21]. Like studies using purified bacterial products, infection studies revealed that direct DC activation by Salmonella also influences phenotypic and functional DC maturation. However, differences in bacterial killing by DCs lacking TLR pathways that were used to remove the effect of pro-inflammatory cytokines (and thus indirect DC maturation) underscores the complexity of mechanistic studies in infection models [21]. Thus, DCs respond differently to direct bacterial contact relative to sensing cytokines in the environment. However, we know little about the role of direct versus indirect DC maturation during infection with a live pathogen, and given the complexity of studying cellular responses during infection with a replicating pathogen, understanding direct versus indirect DC maturation during infection requires further investigation.

\section{Recruitment and Activation of Inflammatory Monocytes and Neutrophils}

In contrast to the modest increase in DCs in response to oral Salmonella infection, neutrophils and inflammatory monocytes rapidly infiltrate Peyer's patches and mesenteric lymph nodes $[1,7,11]$. Inflammatory monocytes can be distinguished from conventional CD11chi DCs and other myeloid cells by, for example, their CD11 ${ }^{\text {int }} \mathrm{CD} 11 b^{\text {hi }}$ Ly6 $6 C^{\text {hi }}$ Ly6G ${ }^{\text {low }}$ phenotype. Indeed, the accumulation of these cells in infected tissues results in their numeric dominance relative to neutrophils and DCs in the gut-associated lymphoid tissue during the acute phase of infection $[7,11]$. Although the importance of bacterial uptake and killing by neutrophils during Salmonella infection has been known for some time, inflammatory monocytes are a relatively recently described myeloid lin- 
eage cell whose function during infection is not fully understood. These cells, however, are predominant producers of products important in fighting bacterial infections, particularly TNF- $\alpha$ and iNOS, in infected tissues [1]. This feature also distinguishes them from conventional $\left(C D 11 c^{\text {hi }}\right)$ DCs, which produce neither iNOS nor TNF- $\alpha$ in Salmonella-infected mice [7, 11]. The ability of inflammatory monocytes to produce TNF- $\alpha$, iNOS and IL- $1 \beta$ combined with their ability to take up and kill Salmonella [7], suggests that they contribute to holding initial bacterial replication in check. Although this may very well be the case, inflammatory monocytes are not as efficient at internalizing Salmonella as neutrophils [7]. Specific depletion experiments and cell type-specific ablation of effector functions, such as TNF- $\alpha$ and iNOS production, by inflammatory monocytes are required to fully elucidate their contribution to bacterial killing in vivo.

Another possible function of inflammatory monocytes during infection is to act as antigen-presenting cells. Indeed, inflammatory monocytes have several features that prompted investigation of their ability to activate $\mathrm{T}$ cells. For example, in addition to their ability to take up and kill Salmonella, they also have relatively high expression of MHC-II and co-stimulatory molecules, particularly CD80 $[7,11]$. These are all are important features of antigen-presenting cells. In addition, inflammatory monocytes express the integrin CD11c at intermediate levels, suggesting a phenotypic, and possibly functional, relationship to CD11chi conventional DCs. However, experimental data show that infection-induced Ly6 $\mathrm{C}^{\text {hi }}$ inflammatory monocytes have a poor capacity to stimulate $\mathrm{T}$ cell proliferation when exposed to Salmonella ex vivo in an antigen-specific system $[7,22]$. Inflammatory monocytes can, however, induce $\mathrm{T}$ cell proliferation when exposed to peptide and can stimulate T cell proliferation in a mixed lymphocyte reaction $[1,7]$. Overall, the data thus far suggest a more likely role of inflammatory monocytes in containing bacterial replication rather than making a major contribution to activating bacteriaspecific $\mathrm{T}$ cells during infection. Indeed, inflammatory monocytes may even inhibit or alter $\mathrm{T}$ cell function [33].

\section{Mechanisms Driving Cell Recruitment to Intestinal Tissues during Infection}

The inflammatory monocytes and neutrophils that accumulate in Salmonella-infected tissues described above are released into the blood early during the response to infection [7]. These cells enter Peyer's patches by a mech- anism that can occur independently of the selectin MAdCAM-1 [7], which is required for T cell homing into Peyer's patches. Insight into the signals that drive monocyte and neutrophil homing to Peyer's patches and MLN during infection were provided by analyzing chemokine receptor expression on cells released into the blood in response to infection combined with kinetic studies of Salmonella-induced chemokine expression in Peyer's patches and MLN $[7,17]$. Not surprisingly, the majority of circulating neutrophils expressed CXCR2 while circulating monocytes expressed a high level of CCR2 [7]. Consistent with this, CXCL2 and CCL2, the ligands for CXCR2 and CCR2, respectively, were significantly upregulated in blood, Peyer's patches and MLN already $48 \mathrm{~h}$ after infection [17]. Another chemokine that can participate in monocyte recruitment, CXCL9, was also upregulated rapidly in gut-associated lymphoid tissue after oral Salmonella infection. Interestingly, the follicle-associated epithelium and cells in the subepithelial dome were the primary initial sources of the neutrophil-recruiting ligand CXCL2. Consistent with their location, the ligand for attracting CCR $6{ }^{+}$DCs to the subepithelial dome in response to Salmonella infection, CCL20, is also predominately produced by the follicle-associated epithelium $[6,17]$. In contrast, localized production of the monocyte-recruiting chemokine CCL2 in Peyer's patches was not readily detected and instead an important source of CCL2 was the myeloid cells themselves in response to bacterial signals [17].

Triggering of pattern recognition receptors by their ligands is important in initiating a large number of events critical to fighting infection, and indeed TLR signaling and the inflammasome are important in controlling Salmonella infection $[30,31]$. To further understand TLR signaling in the innate defense against Salmonella, the role of TLR signaling in myeloid cell recruitment and chemokine production was assessed in Salmonella-infected mice lacking one or more TLRs [17]. These studies revealed that complementarity in the TLR signaling pathways ensures recruitment of myeloid cells to infected tissues. That is, simultaneous removal of both MyD88 and TLR4, whose combined absence removes bacteria-mediated TLR signaling, was required to significantly decrease both monocyte and neutrophil influx into Peyer's patches and MLN. In contrast, removing signaling through TLR4 alone, removing flagella-mediated signaling by infecting wild-type mice with flagella-deficient Salmonella, or infecting TLR4 knockout mice with flagella-deficient Salmonella had no effect on monocyte or neutrophil recruitment to gut-associated lymphoid tissue, while MyD88 deficiency alone partially affected re- 
cruitment [17]. In contrast, release of monocytes and neutrophils from the bone marrow into the blood was not significantly reduced even in infected MyD88 ${ }^{-/-} \mathrm{TLR}^{-/-}$ mice [17]. The observed myeloid cell release into the blood in infected MyD88 ${ }^{-/-} \mathrm{TLR}^{-/-}$mice, and their reduced recruitment into intestinal lymphoid tissues in these animals, occurred despite severe reductions in chemokine expression in the tissues. This suggests that non-TLR-mediated mechanisms such as NLRs may contribute to the release of monocytes and neutrophils into the blood during infection and to the low level of recruitment into infected tissues. Alternatively, low TLR-mediated chemokine levels and/or redundant function of chemokines is sufficient to cause bone marrow release and low recruitment of myeloid cells into tissues during Salmonella infection. The signaling pathways and chemokines required to induce precursor release from the bone marrow, as well as those required for cell influx into infected tissue, are interesting areas that warrant further study.

Overall, TLR signaling is important from the host's perspective to survive infection. Given that a rapid and efficient response is required to contain infections, it is not unexpected that several complimentary and overlapping mechanisms, such as multiple receptors in the same family and distinct receptor families, are in place to ensure host survival. One such example is the multiple TLRs that sense bacterial infection and contribute to phagocyte recruitment to infected lymphoid tissues as described above. Such a finding is not surprising given the importance of recruited phagocytes in limiting bacterial replication and spread. Indeed, mice with defects in TLR signaling, and a corresponding impaired innate immune response, are more susceptible to infection $[17,31]$. However, TLR signaling can also be exploited by Salmonella to enhance virulence. For example, Salmonella-induced TLR4 signaling impairs expression of homeostatic chemokines involved in organizing lymph node architecture, which in turn reduces the efficiency of the host's adaptive immune response and compromises bacterial clearance [34]. Furthermore, TLR signaling is necessary for acidification of Salmonella-containing vacuoles, which is required for induction of Salmonella pathogenicity island II and Salmonella pathogenicity island II-regulated virulence genes [35] that are critical for intracellular survival of Salmonella. That is, in the absence of TLR signaling (in mice with an Nramp-1-resistant background), Salmonella could not replicate intracellularly and thus had reduced virulence [35]. These recent data support a role for TLR signaling in Salmonella virulence, which adds a new layer to the role that TLRs play in host survival.

\section{Concluding Remarks}

Murine infection models have provided much insight into the initial events that occur after Salmonella infection and have paved the way for mechanistic studies allowing detailed understanding of the innate immune response to this oral pathogen. Indeed, recent elegant studies have shed light into the pathways of Salmonella penetration from the intestinal lumen and dissemination in the host, which have been experimentally challenging issues to address. Moreover, we are beginning to get a picture of the orchestration of different myeloid cell populations in responding to Salmonella infection, the mechanisms of their recruitment, and how they contribute to controlling bacterial replication and initiating adaptive immunity. However, there are still gaps in our knowledge, such as the mechanisms of functional DC maturation in vivo and how TLRs as well as other pattern recognition receptors contribute to recruitment and activation of innate cell populations. Moreover, despite a successful journey thus far in building a picture of how the host responds to combat Salmonella, this bacterium continues to reveal surprises. For example, recent studies have revealed how Salmonella exploits TLR signaling to reduce the host's adaptive immune response by impairing expression of chemokines involved in organizing lymph node architecture. TLR signaling is also required for Salmonella to express virulence genes when it resides in phagocytic vacuoles. Thus, the TLR system, which has traditionally been considered a host defense mechanism, also contributes to Salmonella virulence. This new twist of Salmonella pathogenesis highlights that the last word is not said regarding our understanding of the innate immune response to this formidable intracellular pathogen.

\section{Acknowledgements}

The author is grateful to the valuable contributions of past and present group members for their work contributing to the understanding of the innate immune response to Salmonella. Work from the author's group is funded by grants from the Swedish Research Council (621-2007-6536; 621-2010-5238) and the Sahlgrenska Academy at the University of Gothenburg. It was performed at the Mucosal Immunobiology and Vaccine Center (MIVAC) funded by the Swedish Foundation for Strategic Research. 


\section{References}

1 Tam MA, Rydström A, Sundquist M, Wick MJ: Early cellular responses to Salmonella infection: dendritic cells, monocytes and more. Immunol Rev 2008;225:140-162.

$>2$ Halle S, Bumann D, Herbrand H, Willer Y, Dahne S, Forster R, Pabst O: Solitary intestinal lymphoid tissue provides a productive port of entry for Salmonella enterica serovar Typhimurium. Infect Immun 2007;75:15771585.

$>3$ Hapfelmeier S, Muller AJ, Stecher B, Kaiser P, Barthel M, Endt K, Eberhard M, Robbiani R, Jacobi CA, Heikenwalder M, Kirschning C, Jung S, Stallmach T, Kremer M, Hardt WD: Microbe sampling by mucosal dendritic cells is a discrete, MyD88-independent step in $\Delta$ invG $S$. typhimurium colitis. J Exp Med 2008;205:437-450.

$\checkmark 4$ Varol C, Zigmond E, Jung S: Securing the immune tightrope: Mononuclear phagocytes in the intestinal lamina propria. Nat Rev Immunol 2010;10:415-426.

$\checkmark 5$ Schulz O, Jaensson E, Persson EK, Liu X, Worbs T, Agace WW, Pabst O: Intestinal $\mathrm{CD}_{103}{ }^{+}$, but not $\mathrm{CX} 3 \mathrm{CR} 1^{+}$, antigen sampling cells migrate in lymph and serve classical dendritic cell functions. J Exp Med 2009;206: 3101-3114.

6 Iwasaki A, Kelsall BL: Localization of distinct Peyer's patch dendritic cell subsets and their recruitment by chemokines macrophage inflammatory protein (MIP)- $3 \alpha$, MIP-3 $\beta$, and secondary lymphoid organ chemokine. J Exp Med 2000;191:1381-1393.

$>7$ Rydström A, Wick MJ: Monocyte recruitment, activation and function in the gut-associated lymphoid tissue during oral Salmonella infection. J Immunol 2007;178:57895801.

$>8$ Wick MJ: Living in the danger zone: Innate immunity to Salmonella. Curr Opin Micro biol 2004;7:51-57.

$>9$ Kelsall BL, Strober W: Distinct populations of dendritic cells are present in the subepithelial dome and $\mathrm{T}$ cell regions of the murine peyer's patch. J Exp Med 1996;183:237-247.

10 McSorley SJ, Asch S, Costalonga M, Reinhardt RL, Jenkins C: Tracking Salmonellaspecific CD4 $\mathrm{T}$ cells in vivo reveals a local mucosal response to a disseminated infection. Immunity 2002;16:365-377.

11 Sundquist M, Wick MJ: TNF-1-dependent and-independent maturation of dendritic cells and recruited $\mathrm{CD} 11 \mathrm{c}^{\text {int }} \mathrm{CD} 11 \mathrm{~b}^{+}$cells during oral Salmonella infection. J Immunol 2005; 175:3287-3298
12 Voedisch S, Koenecke C, David S, Herbrand $\mathrm{H}$, Forster R, Rhen M, Pabst O: Mesenteric lymph nodes confine dendritic cell-mediated dissemination of Salmonella enterica serovar Typhimurium and limit systemic disease in mice. Infect Immun 2009;77:31703180 .

13 Milling S, Yrlid U, Cerovic V, MacPherson G: Subsets of migrating intestinal dendritic cells. Immunol Rev 2010;234:259-267.

14 Vasquez-Torres A, Jones-Carson J, Bäumler AJ, Falkow S, Valdivia R, Brown W, Le M, Berggren R, Parks WT, Fang FC: Extraintestinal dissemination of Salmonella by CD18expressing phagocytes. Nature 1999;401: 804-808.

15 Worley MJ, Nieman GS, Geddes K, Heffron F: Salmonella typhimurium disseminates within its host by manipulating the motility of infected cells. Proc Natl Acad Sci USA 2006;103:17915-17920.

16 Macpherson AJ, Uhr T: Induction of protective IgA by intestinal dendritic cells carrying commensal bacteria. Science 2004;303: 1662-1665.

17 Rydström A, Wick MJ: Monocyte and neutrophil recruitment during oral Salmonella infection is driven by MyD88-derived chemokines. Eur J Immunol 2009;39:30193030 .

18 Salazar-Gonzalez RM, Niess JH, Zammit DJ, Ravindran R, Srinivasan A, Maxwell JR, Stoklasek T, Yadav R, Williams IR, Gu X, McCormick BA, Pazos MA, Vella AT, Lefrancois L, Reinecker HC, McSorley SJ: CCR6-mediated dendritic cell activation of pathogen-specific T cells in Peyer's patches. Immunity 2006;24:623-632.

19 Reis e Sousa C: Dendritic cells in a mature age. Nat Rev Immunol 2006;6:476-483.

20 Coombes JL, Powrie F: Dendritic cells in instestinal immune regulation. Nat Rev Immunol 2008;8:435-446.

21 Tam M, Sundquist M, Wick MJ: MyD88 and IFN- $\alpha \beta$ differentially control maturation of bystander but not Salmonella-associated dendritic cells or CD11c ${ }^{\text {int }} \mathrm{CD} 11 \mathrm{~b}^{+}$cells during infection. Cell Microbiol 2008;10:15171529.

22 Sundquist M, Wick MJ: Salmonella induces death of $\mathrm{CD} 8 \alpha^{+}$dendritic cells but not CD $11 c^{\text {int }} \mathrm{CD} 11 \mathrm{~b}^{+}$inflammatory cells in vivo via MyD88 and TNFR1. J Leukoc Biol 2009;85: 225-234

23 Shortman K, Heath WR: The $\mathrm{CD}^{+}$dendritic cell subset. Immunol Rev 2010;234:18-31.
24 Hess J, Ladel C, Miko D, Kaufmann SHE: Salmonella typhimurium aroa infection in gene-targeted immunodeficient mice. Major role of CD $4{ }^{+}$TCR- $\alpha \beta$ and IFN- $\gamma$ in bacterial clearance independent of intracellular location. J Immunol 1996;156:3321-3326.

25 Elinav E, Strowig T, Henao-Mejia J, Flavell RA: Regulation of the antimicrobial response by NLR proteins. Immunity 2011;34: 665-679.

26 Kawai T, Akira S: The role of pattern-recognition receptors in innate immunity: update on Toll-like receptors. Nat Immunol 2010;11: 373-384.

-27 Robinson MJ, Sancho D, Slack EC, LeibundGut-Landmann S, Reis e Sousa C: Myeloid C-type lectins in innate immunity. Nat Immunol 2006;7:1258-1265.

28 Trinchieri G, Sher A: Cooperation of Tolllike receptor signals in innate immune defence. Nat Rev Immunol 2007;7:179-190.

29 Davis BK, Wen H, Ting JP: The inflammasome NLRs in immunity, inflammation, and associated diseases. Annu Rev Immunol 2011;29:707-735.

30 Broz P, Newton K, Lamkanfi M, Mariathasan S, Dixit VM, Monack DM: Redundant roles for inflammasome receptors Nlrp3 and Nlrp4 in host defense against Salmonella. J Exp Med 2010;207:1745-1755.

31 Weiss DS, Raupach B, Takeda K, Akira S, Zychlinsky A: Toll-like receptors are temporally involved in host defense. J Immunol 2004; 172:4463-4469.

32 Joffre O, Nolte MA, Sporri R, Reis e Sousa C Inflammatory signals in dendritic cell activation and the induction of adaptive immunity. Immunol Rev 2009;227:234-247.

33 Delano MJ, Scumpia PO, Weinstein JS, Coco D, Nagaraj S, Kelly-Scumpia KM, O’Malley KA, Wynn JL, Antonenko S, Al-Quran SZ, Swan R, Chung CS, Atkinson MA, Ramphal R, Gabrilovich DI, Reeves WH, Ayala A, Phillips J, Laface D, Heyworth PG, ClareSalzler M, Moldawer LL: MyD88-dependent expansion of an immature GR $-1^{+} \mathrm{CD} 11 \mathrm{~b}^{+}$ population induces $\mathrm{T}$ cell suppression and TH2 polarization in sepsis. J Exp Med 2007; 204:1463-1474.

34 St John AL, Abraham SN: Salmonella disrupts lymph node architecture by TLR4-mediated suppression of homeostatic chemokines. Nat Med 2009;15:1259-1265.

35 Arpaia N, Godec J, Lau L, Sivick KE, McLaughlin LM, Jones MB, Dracheva T, Peterson SN, Monack DM, Barton GM: TLR signaling is required for Salmonella typhimurium virulence. Cell 2011;144:675688. 